\title{
Efeito do Ajustamento da Produção de Leite para Período de Serviço Anterior sobre os Valores Genéticos de Bovinos da Raça Holandesa
}

\author{
Nilson Milagres Teixeira1, William José Ferreira ${ }^{2}$, Ary Ferreira de Freitas ${ }^{1}$, \\ Marcus Cordeiro Durães ${ }^{3}$, Márcio Nery Magalhães Junior ${ }^{4}$
}

\begin{abstract}
RESUMO - Registros de produção e reprodução de 6.877 lactações de 4.673 vacas foram usados para se determinar o efeito do ajustamento para período de serviço anterior em avaliações genéticas. Os valores genéticos previstos para produção de leite em kg foram calculados usando-se três modelos: Modelo 1 - sem correção para período de serviço, com os efeitos fixos de rebanho-ano-estação de parto, grupo genético (1-PO, 2-PC), idade ao parto como covariável até $2^{\circ}$ grau e os efeitos aleatórios de animal, meio permanente e erro; Modelo 2 - mesmo anterior com a inclusão de período de serviço anterior; Modelo 3 - mesmo que o modelo 1, com as produções padronizadas para 100 dias de período de serviço anterior, por meio de fatores de ajustamento. Os valores genéticos previstos obtidos para cada modelo foram comparados. Vacas entre as $2 \%$ melhores apresentaram, em média, mudanças de nove e onze posições, respectivamente, quando os modelos 2 e 3 foram usados, comparadas com suas ordens obtidas com o modelo 1. Além disso, oito e onze vacas foram excluídas da relação das $2 \%$ melhores quanto aos seus valores genéticos para produção de leite, quando avaliadas, respectivamente, pelos modelos 2 e 3 . As confiabilidades dos valores genéticos previstos obtidos usando-se os três modelos foram semelhantes. As correlações de ordem entre os valores genéticos estimados, tanto para vacas quanto para touros, para todos os modelos foram próximas de um. Portanto, o ajustamento para período de serviço anterior não alterou a ordem dos animais nesta avaliação genética
\end{abstract} na raça Holandesa no Estado de Minas Gerais.

Palavras-chave: ajustamento, período de serviço, produção de leite, raça Holandesa

\section{Effect of Adjusting Production Records for Days Open on Breeding Values in Holsteins}

\begin{abstract}
Production and breeding records from 6,877 lactations of 4,673 cows were used to evaluate the effect of adjusting for previous days open on genetic evaluations. Predicted breeding values for kg of milk were calculated using three animal models. Model 1 - contained no adjustment for days open and accounted for the fixed effects of herd-year-season, genetic group, age of calving (covariate) and the random effects of animal, permanent environment effect and error; Model 2 - same as one with previous days open included; Model 3 - production records were standardized to 100 days open before using model 1 . Predicted breeding values from each model were compared. Cows in the $2 \%$ top had their ranks changed nine and 11 places for models 2 and 3 , respectively, when compared to their rank from model 1. Furthermore, eight and 11 cows dropped from the top $2 \%$, when evaluated with models 2 and 3 , both accounted for days open. The reliabilities of the predicted breeding values for the three models were almost equal. The rank correlations between estimated breeding values for cows and sires for all models were near one. Therefore, adjustment for previous days open did not change the rank of animals in this genetic evaluation for Holsteins in the State of Minas Gerais.
\end{abstract}

Key Words: adjustment, days open, milk yield, Holstein breed

\section{Introdução}

Nas avaliações genéticas em gado de leite, espera-se que as ordenações dos animais quanto aos seus valores genéticos sejam corretas e as previsões da magnitude das diferenças genéticas entre dois animais quaisquer, as mais precisas possíveis. Nas avaliações na raça Holandesa do Estado de Minas Gerais, pelo terceiro ano consecutivo, vem-se utilizando um sistema com base no modelo animal, em que se estimam valores genéticos para leite e gordura (TEIXEIRA et al., 1998). Pressupõe-se que o modelo estatístico, ora em uso, é o melhor que se ajusta aos dados, isto é, que contempla o maior número possível de efeitos que interferem na variação da produção, possibilitando comparações entre os animais com eliminação do máximo de diferenças não-genéticas. O período de serviço e o intervalo de partos são fatores de meio ambiente que precisam ser considerados em avaliações genéticas para se obterem va-

\footnotetext{
${ }_{1}$ Pesquisador da Embrapa Gado de Leite e Bolsista do CNPq. E.mail: nilson@cnpgl.embrapa.br; ary@cnpgl.embrapa.br

2 Estudante de Pós-Graduação da UFV. E.mail: william@alunos.ufv.br

3 Pesquisador da Embrapa Gado de Leite. E.mail: dcm075@cnpgl.embrapa.br

4 Técnico da Associação de Criadores de Gado Holandês de Minas Gerais.
} 
lores genéticos mais precisos para características produtivas (METZGER et al., 1990; SADEK e FREEMAN, 1992; TEIXEIRA et al., 1999). Em 12 países dos 28 participantes de avaliação internacional, ocorre o ajustamento para a relação entre reprodução e produção nas avaliações genéticas para características produtivas (INTERBULL, 1992). A partir de janeiro de 1995, nos Estados Unidos, os registros de produção e os valores genéticos passaram a ser ajustados para período de serviço na lactação anterior, portanto, as produções nas primeiras lactações não são ajustadas (VANRADEN et al., 1995).

A períodos de serviços curtos estão associadas menores produções durante o período final da lactação, uma vez que os nutrientes ingeridos naquele período serão compartilhados para produção e gestação, podendo também resultar em lactações mais curtas do que 305 dias (LOUCA e LEGATES, 1968). Por outro lado, havendo mais dias de período de serviço, haverá mais tempo para renovação das reservas corporais, que poderão ser usadas na lactação seguinte. A produção durante as segundas e lactações seguintes foram influenciadas pelo período de serviço anterior além do período de serviço corrente (FUNK et al., 1987; SADEK e FREEMAN, 1992; TEIXEIRA et al., 1999). Fatores de ajustamento são usados para remover diferenças de meio ambiente entre os animais. Valores estimados da heritabilidade do período de serviço e do intervalo de partos têm sido geralmente baixos e inferiores a 0,08 , uma indicação de serem características essencialmente influenciadas por diferenças de meio ambiente (HANSEN et al., 1983; FUNK et al., 1987; TEIXEIRA et al., 1999). Contudo, a produção de leite deve ser ajustada para período de serviço somente se tiver efeito sobre a produção. Efeitos significativos foram relatados em vários trabalhos (OLTENACU et al., 1980; THOMPSON et al., 1982; FUNK et al., 1987; SADEK e FREEMAN, 1992; TEIXEIRA et al., 1999). TEIXEIRA et al. (1999), utilizando modelo de touro, sugeriram fatores de ajustamento da produção de leite para período de serviço anterior e corrente e concluíram ser conveniente o ajustamento da produção de leite para período de serviço, ao se estimarem valores genéticos de touros.

Uma vez que o período de serviço anterior pode, quase sempre, ser determinado pelas datas de parto das lactações anterior e corrente, para vacas individuais, e a inclusão do período de serviço corrente implica em redução acentuada do número de registros, com este trabalho, procurou-se verificar o efeito de alternativas de ajustamento para período de serviço anterior sobre os valores genéticos previstos para vacas e touros obtidos usando-se modelo animal.

\section{Material e Métodos}

Utilizaram-se registros de produção e reprodução obtidos do Serviço de Controle Leiteiro da Associação dos Criadores de Gado Holandês de Minas Gerais. Os períodos de serviço foram calculados subtraindo-se 283 dias de intervalos de partos consecutivos. As produções de leite analisadas referiram-se a lactações normais e iniciadas em rebanhos-anos com no mínimo 20 lactações com duração de 120 a 450 dias, de vacas com primeira lactação conhecida. A produção de leite até 305 dias foi ajustada para duas ordenhas, conforme TEIXEIRA (1998). Os touros deveriam ter pelo menos cinco filhas em pelo menos três rebanhos e cada classe rebanho-ano-estação de parto filhas de pelo menos dois touros. Foram usadas 6877 lactações de 4673 vacas de segundas ou lactações seguintes, satisfazendo as restrições anteriores e com partos no período de 1992 e 1997. Um arquivo de "pedigree" com a identificação da vaca, pai e mãe de 46.369 animais, resultou em 62.885 indivíduos na matriz de parentesco usada em todas as análises com o modelo animal.

Os valores genéticos previstos para produção de leite até 305 dias foram obtidos usando-se três modelos animal. No primeiro (MOD01), não se considerou o período de serviço, ajustando-se os efeitos fixos de rebanho-ano-estação, grupo genético (1-Puras de origem, 2-Puras por cruza), efeito da idade ao parto como covariável até $2^{\circ}$ grau e os efeitos aleatórios de animal, meio ambiente permanente e erro. As estações de parto consideradas foram: águas, de outubro a março e seca, de abril a setembro. No segundo modelo (MOD02), todos os efeitos anteriores foram considerados, incluindo-se também o período de serviço anterior. O terceiro modelo (MOD03) foi o mesmo MOD01, com a diferença de que as produções foram previamente padronizadas para 100 dias de período de serviço anterior, por meio de fatores de ajustamento, sugeridos por TEIXEIRA et al. (1999). O número de classes rebanho-ano-estação representadas foi de 1.210. Os valores genéticos de vacas e touros, obtidos por meio do sistema MTDFREML (BOLDMAN et al., 1995), foram comparados por intermédio de correlações de ordem. 


\section{Resultados e Discussão}

As médias, os desvios-padrão e coeficientes de variação para produção de leite até 305 dias foram, respectivamente, $6221,7 \mathrm{~kg}, 1944,5 \mathrm{~kg}$ e $31,4 \%$ e para período de serviço 124,2 dias, 63,9 dias e 51,42\%.

As estimativas dos componentes de variância e das heritabilidades da produção de leite até 305 dias, obtidas usando-se os três modelos propostos, foram semelhantes (Tabela 1). As médias e os respectivos desvios-padrão das confiabilidades dos valores genéticos para produção de leite das 100 melhores vacas e dos 10 melhores touros, obtidos pelos três modelos de análise, não diferiram e foram, respecti- vamente, $0,59 \pm 0,06$ e $0,71 \pm 0,14$. As correlações de ordem entre os valores genéticos para vacas e touros estimadas pelos três modelos foram altas. Os valores genéticos obtidos pelo MOD01 e MOD02 foram 0,97 e 0,96 , respectivamente, para vacas e touros e os obtidos pelo MOD01 e MOD03, 0,96 e 0,97. O número médio de mudanças de ordem e o número de animais excluídos usando-se MOD02 e MOD03 para diferentes porcentagens de vacas e touros selecionados pelo modelo 1 encontram-se na Tabela 2. Com o aumento da proporção selecionada de vacas, houve redução no número médio de mudanças de ordem. No caso dos touros, com o aumento da proporção selecionada, ocorreu maior número de trocas de posição.

Tabela 1 - Componentes de variância e heritabilidades da produção de leite até 305 dias usando-se três diferentes modelos animal

Table 1 - Variance components and heritabilities of 305-days milk yield using three animal models

\begin{tabular}{|c|c|c|c|}
\hline $\begin{array}{l}\text { Estimativa } \\
\text { Estimate }\end{array}$ & MOD01 $^{1}$ & $\mathrm{MOD}^{2} 2^{2}$ & ${\mathrm{MOD} 03^{3}}^{3}$ \\
\hline \multicolumn{4}{|l|}{ Variância } \\
\hline \multicolumn{4}{|l|}{ Variance } \\
\hline Genética & $509.947,8766$ & $510.870,4799$ & $513.469,2875$ \\
\hline \multicolumn{4}{|l|}{ Genetic } \\
\hline Ambiente & $1.181 .125,0118$ & $1.172 .423,1311$ & $1.173 .052,0965$ \\
\hline \multicolumn{4}{|l|}{ Environmental } \\
\hline Ambiente-Permanente & $415.111,0000$ & $393.957,0000$ & $389.451,0000$ \\
\hline \multicolumn{4}{|l|}{ Permanent environment } \\
\hline Fenotípica & $2.106 .184,1365$ & $2.077 .250,9923$ & $2.075 .972,5698$ \\
\hline \multicolumn{4}{|l|}{ Phenotypic } \\
\hline Heritabilidade & 0,24 & 0,25 & 0,25 \\
\hline Heritability & & & \\
\hline
\end{tabular}

Tabela 2 - Número médio de mudanças de ordem e número de animais excluídos usando-se modelos 2 e 3 para diferentes porcentagens de vacas e touros selecionados pelo modelo ${ }^{1}$

Table 2 - Average number of changes of rank and number of animals dropped using models 2 and 3 from different percentages of cows and sires selected using model ${ }^{1}$

\begin{tabular}{|c|c|c|c|c|}
\hline \multirow{2}{*}{$\begin{array}{l}\% \text { selecionada } \\
\% \text { selected }\end{array}$} & \multicolumn{2}{|c|}{ MOD02 } & \multicolumn{2}{|c|}{ MOD03 } \\
\hline & $\begin{array}{c}\text { Mudanças } \\
\text { Changes }\end{array}$ & $\begin{array}{c}\text { Excluídos } \\
\text { Dropped }\end{array}$ & $\begin{array}{c}\text { Mudanças } \\
\text { Change }\end{array}$ & $\begin{array}{c}\text { Excluídos } \\
\text { Dropped }\end{array}$ \\
\hline \multicolumn{5}{|l|}{ Vacas 1} \\
\hline \multicolumn{5}{|l|}{ Cows ${ }^{1}$} \\
\hline 1 & 12,0 & 3,0 & 15,0 & 3,0 \\
\hline 2 & 9,0 & 8,0 & 11,0 & 11,0 \\
\hline 3 & 6,0 & 7,0 & 8,0 & 8,0 \\
\hline 5 & 4,0 & 12,0 & 5,0 & 12,0 \\
\hline \multirow{2}{*}{\multicolumn{5}{|c|}{$\begin{array}{l}\text { Touros } \\
\text { Sires }^{2}\end{array}$}} \\
\hline & & & & \\
\hline 5 & 1,0 & 0,0 & 1,0 & 1,0 \\
\hline 10 & 2,0 & 1,0 & 2,0 & 0,0 \\
\hline 20 & 3,0 & 4,0 & 3,0 & 5,0 \\
\hline 30 & 4,0 & 6,0 & 4,0 & 5,0 \\
\hline
\end{tabular}

14673 vacas com produção conhecida; ${ }^{2} 313$ touros avaliados.

14673 cows with known milk production; 2313 sires evaluated. 
Vacas entre as $2 \%$ melhores quanto aos seus valores genéticos pelo MOD01 apresentaram, em média, mudanças de nove e onze posições, respectivamente, quando MOD02 e MOD03 foram usados, comparadas com suas ordens obtidas com MOD01. À medida que se aumentou o número de animais selecionados, houve tendência de aumento de animais excluídos, de modo semelhante com o uso de MOD02 e MOD03. TEIXEIRA et al. (1999) analisaram dados de rebanhos das Regiões Sul e Sudeste do Brasil e utilizaram modelo de touro. Esses autores compararam valores genéticos dos touros para produção de leite até 305 dias, ajustada à maturidade, de 10.708 lactações, quando os períodos de serviço anterior e corrente foram incluídos e(ou) excluídos nos modelos de análise, encontrando correlações de ordem entre os valores genéticos de touros também próximos de um. A despeito dos resultados, argumentaram que o ajustamento afetou os valores genéticos, bem como removeu os efeitos de meio ambiente atribuído a período de serviço, sobre a produção. Neste trabalho, não houve alteração das confiabilidades

Tabela 3 - Ordem de classificação, valor genético (VG) e confiabilidade (Conf.) de vacas e touros de acordo com o modelo de análise

Table 3 - Rank, breeding value (BV) and reliability (Rel.) for cows and sires according to model of analysis

\begin{tabular}{|c|c|c|c|c|c|c|c|c|c|}
\hline \multirow[b]{2}{*}{$\begin{array}{l}\text { Número } \\
\text { Number }\end{array}$} & \multicolumn{3}{|c|}{ MOD01 } & \multicolumn{3}{|c|}{ MOD02 } & \multicolumn{3}{|c|}{ MOD03 } \\
\hline & $\begin{array}{c}\text { Ordem } \\
\text { Rank }\end{array}$ & $\begin{array}{l}\mathrm{VG} \\
B V\end{array}$ & $\begin{array}{l}\text { Conf. } \\
\text { Rel. }\end{array}$ & $\begin{array}{c}\text { Ordem } \\
\text { Rank }\end{array}$ & $\begin{array}{l}\mathrm{VG} \\
B V\end{array}$ & $\begin{array}{c}\text { Conf. } \\
\text { Rel. }\end{array}$ & $\begin{array}{c}\text { Ordem } \\
\text { Rank }\end{array}$ & $\begin{array}{l}\mathrm{VG} \\
B V\end{array}$ & $\begin{array}{c}\text { Conf. } \\
\text { Rel. }\end{array}$ \\
\hline \multicolumn{9}{|c|}{ Vacas } & \\
\hline 61580 & 1 & 1612 & 0,63 & 1 & 1636 & 0,63 & 1 & 1628 & 0,63 \\
\hline 52924 & 2 & 1609 & 0,64 & 2 & 1615 & 0,65 & 2 & 1625 & 0,65 \\
\hline 42710 & 3 & 1466 & 0,64 & 4 & 1465 & 0,64 & 4 & 1487 & 0,65 \\
\hline 55930 & 4 & 1437 & 0,65 & 3 & 1493 & 0,66 & 3 & 1538 & 0,66 \\
\hline 60673 & 5 & 1409 & 0,61 & 5 & 1425 & 0,61 & 5 & 1433 & 0,61 \\
\hline 51462 & 6 & 1349 & 0,57 & 7 & 1365 & 0,58 & 7 & 1350 & 0,58 \\
\hline 62150 & 7 & 1333 & 0,60 & 9 & 1328 & 0,60 & 10 & 1324 & 0,60 \\
\hline 57590 & 8 & 1326 & 0,60 & 6 & 1373 & 0,61 & 6 & 1372 & 0,61 \\
\hline 52933 & 9 & 1281 & 0,57 & 12 & 1284 & 0,58 & 14 & 1250 & 0,58 \\
\hline 52774 & 10 & 1276 & 0,66 & 8 & 1339 & 0,66 & 8 & 1336 & 0,67 \\
\hline 61787 & 11 & 1273 & 0,58 & 13 & 1282 & 0,58 & 13 & 1266 & 0,58 \\
\hline 39082 & 12 & 1272 & 0,57 & 10 & 1313 & 0,57 & 9 & 1326 & 0,58 \\
\hline 56133 & 13 & 1257 & 0,59 & 11 & 1303 & 0,59 & 11 & 1309 & 0,59 \\
\hline 62148 & 14 & 1257 & 0,59 & 16 & 1249 & 0,59 & 18 & 1230 & 0,59 \\
\hline 60513 & 15 & 1242 & 0,58 & 18 & 1246 & 0,58 & 15 & 1241 & 0,58 \\
\hline 62011 & 16 & 1232 & 0,58 & 19 & 1234 & 0,58 & 19 & 1218 & 0,58 \\
\hline 52175 & 17 & 1227 & 0,65 & 15 & 1252 & 0,65 & 17 & 1233 & 0,65 \\
\hline 55613 & 18 & 1215 & 0,60 & 20 & 1190 & 0,60 & 26 & 1153 & 0,61 \\
\hline 55495 & 19 & 1209 & 0,64 & 14 & 1265 & 0,64 & 12 & 1278 & 0,64 \\
\hline 57740 & 20 & 1189 & 0,64 & 17 & 1249 & 0,64 & 16 & 1240 & 0,64 \\
\hline 50953 & 21 & 1181 & 0,59 & 28 & 1137 & 0,59 & 27 & 1142 & 0,60 \\
\hline 48248 & 22 & 1176 & 0,60 & 23 & 1186 & 0,61 & 23 & 1175 & 0,61 \\
\hline 53297 & 23 & 1173 & 0,61 & 31 & 1134 & 0,61 & 34 & 1118 & 0,61 \\
\hline 44024 & 24 & 1164 & 0,64 & 26 & 1144 & 0,65 & 32 & 1125 & 0,65 \\
\hline 57616 & 25 & 1159 & 0,54 & 50 & 1069 & 0,54 & 49 & 1068 & 0,54 \\
\hline \multicolumn{10}{|c|}{$\begin{array}{c}\text { Touros } \\
\text { Sires }\end{array}$} \\
\hline 1284 & 1 & 1704 & 0,89 & 1 & 1717 & 0,89 & 1 & 708 & 0,89 \\
\hline 1477 & 2 & 1358 & 0,83 & 2 & 1423 & 0,84 & 2 & 1409 & 0,84 \\
\hline 1389 & 3 & 1277 & 0,63 & 3 & 1280 & 0,63 & 3 & 1267 & 0,63 \\
\hline 1670 & 4 & 1167 & 0,73 & 4 & 1202 & 0,74 & 4 & 1234 & 0,74 \\
\hline 1125 & 5 & 972 & 0,61 & 6 & 970 & 0,62 & 5 & 969 & 0,62 \\
\hline 554 & 6 & 948 & 0,83 & 5 & 982 & 0,83 & 6 & 950 & 0,83 \\
\hline 1143 & 7 & 896 & 0,60 & 8 & 878 & 0,60 & 8 & 888 & 0,60 \\
\hline 1596 & 8 & 884 & 0,62 & 7 & 898 & 0,62 & 7 & 947 & 0,62 \\
\hline 1152 & 9 & 787 & 0,50 & 11 & 769 & 0,50 & 11 & 760 & 0,50 \\
\hline 1525 & 10 & 786 & 0,85 & 10 & 835 & 0,85 & 10 & 846 & 0,85 \\
\hline
\end{tabular}


médias dos valores genéticos e das correlações de ordem. Os valores genéticos das vacas foram mais afetados pelos ajustamentos que os valores genéticos dos touros. Oito $(8,5 \%)$ e onze $(11,8 \%)$ vacas não foram classificadas entre as $93(2 \%)$ melhores quando avaliadas, segundo os modelos 2 e 3 , respectivamente, comparados com a ordenação obtida pelo modelo 1. Na Tabela 3, encontram-se as 25 melhores vacas e os dez melhores touros, conforme MOD01, com suas classificações, valores genéticos e respectivas confiabilidades obtidos pelos três modelos. No caso dos touros, as diferenças entre os modelos são pequenas. O número máximo de excluídos foi seis, quando até $30 \%$ foram selecionados e o período de serviço foi ajustado no modelo de análise. Os valores genéticos das vacas podem ser usados para orientar descartes em rebanhos individuais. Quando o rebanho com maior número de vacas foi analisado e poucas foram descartadas com base nos valores genéticos obtidos pelo MOD01, houve maior variabilidade das porcentagens de vacas em comum usando-se MOD02 e MOD03 (Tabela 4). Entretanto, quando o descarte foi de 20 a $30 \%$ das vacas do rebanho, houve coincidência de, aproximadamente, $97 \%$ das mesmas, usando-se os diferentes modelos. Em vista destes resultados, possivelmente, não se justifica, por enquanto, o ajustamento para período de serviço anterior.

Tabela 4 - Porcentagens de vacas descartadas usando-se MOD01, em comum com MOD02 e MOD03 para porcentagens crescentes de descarte

Table 4 - Percentages of culled cows using MOD01 in common with MOD02 and MODO3 for increasing percentages of culling

\begin{tabular}{lccc}
\hline $\begin{array}{l}\text { Descarte }(\%) \\
\text { Culling }(\%)\end{array}$ & $\begin{array}{c}\text { Vacas }(\mathrm{n}-\mathbf{0}) \\
\text { Cows }(n .)\end{array}$ & $\begin{array}{c}\text { \% de vacas em comum } \\
\text { \% of cows in common }\end{array}$ \\
\cline { 3 - 4 } & & 50 & MOD02 \\
\hline 1 & 2 & 86 & MOD03 \\
\hline 3 & 7 & 100 & 100 \\
5 & 12 & 94 & 100 \\
7 & 17 & 95 & 91 \\
9 & 22 & 96 & 96 \\
10 & 25 & 93 & 97 \\
12 & 30 & 94 & 94 \\
14 & 34 & 92 & 95 \\
15 & 37 & 96 & 98 \\
20 & 49 & 97 & 97 \\
25 & 61 & 97 & 99 \\
30 & 74 & 97 & 98 \\
35 & 86 & 96 & 98 \\
40 & 98 & 96 & 99 \\
45 & 111 & 96 & 97 \\
50 & 123 & & \\
\hline
\end{tabular}

\section{Conclusões}

Os valores genéticos previstos das vacas foram mais afetados pela inclusão do período de serviço anterior no modelo ou o ajustamento prévio das produções para um período de serviço padrão do que os valores genéticos dos touros. Considerando-se que os valores genéticos são atualmente usados para orientar descartes e reposições de vacas nos rebanhos do Estado, possivelmente, por enquanto, não se justifica o ajustamento para período de serviço anterior.

\section{Referências Bibliográficas}

BOLDMAN, K.G., KRIESE, L.A., VAN VLECK, L.D. et al. 1995. A manual for use of MTDFREML . A set of programs to obtain estimates of variances and covariances [Draft]. Beltsville: U.S. Department of Agriculture, Agricultural Research Service. 125p.

FUNK, D.A., FREEMAN, A.E, BERGER, P.J. 1987. Effect of previous days open, previous days dry, and present days open on lactation yield. J.Dairy Sci., 70(11):2366-2373.

HANSEN, L.B., FREEMAN, A.E., BERGER, P.J. 1983. Yield and fertility relationships in dairy cattle. J. Dairy Sci., 66(2):293-305.

INTERBULL, 1992. Sire evaluation procedures for dairy production traits practiced in various countries, Uppsala: Dep. Anim Breed. Genet. SLU( Int. Bull Eval. Serv. Bull. 5).

LOUCA, A., LEGATES, J. E. 1968. Yield losses in dairy cattle due to days open. J. Dairy Sci., 51(6):573-583.

METZGER, J.S., SCHUTZ, M.M., FREEMAN, A.E. 1990. Adjusting production records of days open using an animal model. J. Dairy Sci., 73(Suppl.1):142 (Abstr).

OLTENACU, P.A., ROUNSAVILLE, T.R., MILLIGAN, R.A. et al. 1980. Relationships between days open and cumulative milk yield at various intervals from parturition for high and low producing cows. J. Dairy Sci., 63(8):1317-1327.

PHILIPSSON, J., DOMMERHOLT, J., FIMLAND, E. et al. 1978. Problems in cow evaluation and current use of cow index. Report of a working group on cow evaluation. Livest. Prod. Sci., 5(1):3-18.

SADEK, M.H., FREEMAN, A.E. 1992. Adjustment factors for previous and present days open considering all lactations. J. Dairy Sci., 75(1):279-287.

TEIXEIRA, N.M. 1998. Padronização da produção de leite para duas ordenhas na raça Holandesa. Juiz de Fora: Embrapa Gado de Leite. 1p. (Embrapa Gado de Leite. Folha solta,38).

TEIXEIRA, N.M., FREITAS, A.F., MAGUALHÃES JR., M.N. 1998. PTAs de vacas. Minas de Leite. Juiz de Fora: Associação dos Criadores de Gado Holandês de Minas Gerais, v.4, n.47, p.4.

TEIXEIRA, N.M., VALENTE, J., VERNEQUE, R.S. et al. 1999. Efeito do ajustamento para período de serviço anterior e corrente em avaliações genéticas para produção de leite na raça Holandesa. R. Soc. Bras. Zootec., 28(2):288-294.

THOMPSON, J.R., FREEMAN, A.E., BERGER, P.J. 1982. Days open adjusted, annualized and fat-corrected yields as alternatives to mature equivalent records. J. Dairy Sci., 65(8):1562-1577.

VANRADEN, P.M., WIGGANS, G.R., POWELL, R.L. et al. 1995. Changes in USDA-DHIA genetic evaluations. Beltsville: Animal Improvement Progr. Lab. (AIPL Res. Rep. CH3 (1-95).

Recebido em: 05/09/00 Aceito em: 20/12/00 